Original Article

\title{
Exploring the beneficial role of telmisartan in sepsis-induced myocardial injury through inhibition of high-mobility group box 1 and glycogen synthase kinase-3 $\beta /$ nuclear factor-kB pathway
}

\author{
Yan Jin ${ }^{1}$, Hong Wang ${ }^{1}$, Jing Li ${ }^{2}$, Minyan Dang ${ }^{3}$, Wenzhi Zhang ${ }^{3}$, Yan Lei ${ }^{3}$, and Hao Zhao ${ }^{4, *}$ \\ 'Emergency Department, Second Affiliated Hospital of Dalian Medical University Dalian, Jinan, Shandong 116027, ${ }^{2}$ Department of Cardiology, Hospital \\ Affiliated to Shenyang Medical College, Shenyang, Liaoning 110021, P.R. China, ${ }^{3}$ Innoscience Research Sdn Bhd, Subang Jaya, Selangor 47650, Malaysia, \\ ${ }^{4}$ Emergency Department, Affiliated Hospital of Shandong University of Traditional Chinese Medicine, Jinan, Shandong 250014, P.R. China
}

\section{ARTICLE INFO}

Received January 9, 2020

Accepted January 31,2020

*Correspondence

Hao Zhao

E-mail:zhhaosci7702@sina.com

Key Words

High-mobility group protein

HMGB1

Kinases

Sepsis

Telmisartan

\begin{abstract}
In the present experimental study, cecal ligation and puncture significantly increased the myocardial injury assessed in terms of excess release of creative kinase-MB (CK-MB), cardiac troponin I (cTnl), interleukin (IL)- 6 and decrease of IL-10 in the blood following $12 \mathrm{~h}$ of laparotomy procedure as compared to normal control. Also, a significant increase in protein expression levels of high-mobility group box 1 (HMGB1) and decreased phosphorylation of glycogen synthase kinase-3 $\beta$ (GSK-3 $\beta$ ) was observed in the myocardial tissue as compared to normal control. A single independent administration of telmisartan ( 2 and $4 \mathrm{mg} / \mathrm{kg}$ ) and AR-A014418 (1 and 2 $\mathrm{mg} / \mathrm{kg}$ ) substantially reduced sepsis-induced myocardial injury in terms of decrease levels of CK-MB, cTnl and IL-6, HMGB1, GSK-3 $\beta$ and increase in IL-10 and p-GSK-3 $\beta$ in the blood in sepsis- subjected rats. The effects of telmisartan at dose $4 \mathrm{mg} / \mathrm{kg}$ and AR-A014418 at a dose of $2 \mathrm{mg} / \mathrm{kg}$ were significantly higher than the telmisartan at a dose of $2 \mathrm{mg} / \mathrm{kg}$ and AR-A014418 $1 \mathrm{mg} / \mathrm{kg}$ respectively. Further, no significant effects on different parameters were observed in the sham control group in comparison to normal. Therefore it is plausible to suggest that sepsis may increase the levels of angiotensin II to trigger GSK-3 $\beta$-dependent signaling to activate the HMGB1/receptors for advanced glycation end products, which may promote inflammation and myocardial injury in sepsis-subjected rats.
\end{abstract}

\section{INTRODUCTION}

Sepsis is defined as a systemic inflammatory response syndrome, mainly caused by infection [1] and sepsis-induced myocardial injury is the manifestation of multiple organ failure in sepsis, which is characterized by myocardial injury, ventricular dilatation, reduced contractility, impaired ventricular response $[2,3]$. Indeed, sepsis leads to the initiation of a complex intramyocardial inflammatory response, which results in the development of sepsis-induced myocardial dysfunction. Sepsis-induced myocardial injury is worldwide recognized as a serious major health burden, which significantly accounts for morbidity and mortality [4]. Despite the advances in the understanding of the intramyocardial inflammatory response, there is no promising target to treat this sepsis-induced myocardial dysfunction. Accordingly, there is a need to identify the underlying mechanisms associated with sepsis-induced myocardial injury.

Angiotensin II (Ang II) is an octapeptide peptide, which possesses diverse biological functions including activation of the inflammatory response through different mechanisms $[5,6]$. It is shown to significantly enhance the interleukin-6 (IL-6) production, macrophage recruitment, vascular inflammation and

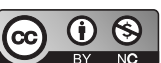

This is an Open Access article distributed under the terms of the Creative Commons Attribution Non-Commercial License, which permits unrestricted non-commercial use, distribution, and reproduction in any medium, provided the original work is properly cited. Copyright (c) Korean J Physiol Pharmacol, pISSN 1226-4512, elSSN 2093-3827
Author contributions: Y.J. Experiment conduct, Manuscript writing; H.W. Statistical analysis and result writing; J.L. Manuscript writing, conduct estimations; M.D. Experiment conduct; W.Z. Estimation analysis; Y.L. Manuscript writing, statistical analysis; H.Z. Study design, analysis. 
remodeling in older C57BL/6J mice [7]. Using transgenic mice TgAng II, it has been found that in cardiac Ang II increases inflammation, oxidative stress, and cell death, which ultimately leads to cardiac fibrosis [8]. Further, the administration of angiotensin receptor $1\left(\mathrm{AT}_{1}\right)$ antagonist has been shown to prevent cardiac and aortic tissue alterations, inflammatory cell infiltration and cytokine as well as metalloproteinase expression, suggesting the involvement of Ang II in the induction of heart and aortic inflammation [5]. However, the role of Ang II in sepsis-induced myocardial injury is not explored yet.

High-mobility group box 1 (HMGB1), an intracellular protein, acts as an early mediator of inflammation and organ damage [6,9]. It exerts its pro-inflammatory actions via binding to receptors for advanced glycation end products (RAGE). HMGB1-RAGE axis has been implicated in several inflammatory heart disorders $[10,11]$. Furthermore, it has also been reported that treatment with angiotensin receptor blockers significantly inhibits the HMGB1/ RAGE axis in stroke and hypertensive patients [12,13]. Since the molecular mechanisms of inflammatory sepsis-induced cardiac injury are not fully understood and considering the HMGB1/ RAGE axis as an important target of inflammatory disorder, the present study was aimed to identify the role HMGB1/RAGE axis in sepsis-induced myocardial injury.

Glycogen synthase kinase-3 (GSK-3) is a serine/threonine protein kinase, which regulates a variety of intracellular signaling pathways and has been implicated in many neuronal and cardiac disorders. A large number of studies have demonstrated the role of GSK- $3 \beta$ isoform in various cardiac pathological conditions including ischemic injury, myocardial fibrosis and cardiomyocyte proliferation $[14,15]$. Also, it has been reported that decreased GSK-3 $\beta$ activity is beneficial in protecting the murine heart against ischemia-reperfusion injury [16]. Furthermore, GSK-3 $\beta$ has been identified as an important mediator of inflammation in diseased conditions. However, the role of GSK-3 $\beta$ in sepsisinduced myocardial injury is unexplored. Therefore, the present study was aimed to explore the role of Ang II and GSK-3 $\beta$ in sepsis-induced myocardial injury using selective pharmacological agents i.e., telmisartan and AR-A014418, respectively along with the underlying mechanism.

\section{METHODS}

\section{Experimental animals and drugs}

Male Wistar albino rats (200-250 g) were employed for this study and were kept in the animal house of the department with standard laboratory conditions i.e., under controlled temperature, humidity, chow diet and the normal cycle of $12 \mathrm{~h}$ light and $12 \mathrm{~h}$ dark. All the experiments were also performed per the Animal Ethics Committee of the Second Affiliated Hospital of Dalian Medical University-wide Ethic number: 2019211. Telmisartan
(Sigma, St. Louis, MO, USA) and AR-A014418 (Cayman Chemical Co., Ann Arbor, MI, USA) were dissolved in normal saline containing 10\% DMSO. IL-6, IL-10, creative kinase-MB (CK-MB) and cardiac troponin I (cTnI) ELISA detection kits were purchased from Beijing Cheng Lin Biotechnology Co., Ltd., Beijing, China. HMGB1 ELISA kit was purchased from MyBioSource, Inc., San Diego, CA, USA for the quantitative determination of HMGB1 in the tissue homogenate. GSK-3 $\beta$ ELISA kit was purchased from Ray Biotech Pvt. Ltd., Norcross, GA, USA for estimation of total GSK-3 $\beta$ and p-GSK3 $\beta$-S9 levels.

\section{Induction of sepsis by cecal ligation puncture}

The rat model of sepsis was established by employing the cecal ligation and puncture (CLP) method as previously described [17]. Intraperitoneal (i.p.) injection of pentobarbital sodium (20 $\mathrm{mg} / \mathrm{kg}$ ) was administered to anesthetize rats $15 \mathrm{~min}$ before the surgery. Under aseptic conditions, the laparotomy was performed and a $2 \mathrm{~cm}$ incision was made to expose the cecum. Afterward, the cecum was ligated with a sterile 3-0 silk suture at its base and the cecum was punctured twice using an 18-gauge needle. Afterward, the cecum was squeezed to extrude some fecal material from the punctured site. Following this, the cecum was returned to the abdominal cavity and incision was closed with silk sutures. Following the laparotomy procedure, all rats were administered with saline ( $3 \mathrm{ml} / 100 \mathrm{~g}$ i.p.) and returned to their cages. Twelve hours after the laparotomy, the rats were sacrificed via cervical dislocation and their abdomens were opened to collect $5 \mathrm{ml}$ blood from the abdominal aorta. The midline sternotomy was performed and the hearts were removed. The blood samples and myocardial tissues were stored at $-80^{\circ} \mathrm{C}$ in the refrigerator. The blood samples were used for the determination of serum CKMB and CTnI levels. All rats underwent CLP surgical procedures except the normal rats. Sham control animals underwent the same surgical procedure, without cecum ligation and puncture.

\section{Biochemical markers of myocardial injury}

The extent of myocardial injury was assessed by determination of serum CKMB and cTnI levels using ELISA diagnostic kits. The CK-MB results were expressed in U/l and cTnI results were expressed in $\mathrm{ng} / \mathrm{ml}$.

\section{Assessment of other biochemical parameters}

The heart tissue was homogenized to quantify the levels of HMGB1, p-GSK-3 and GSK-3 $\beta$ in the myocardial tissue using ELISA kits. The levels of IL- 6 and IL10 were quantified in the blood using specific ELISA diagnostic kits. 


\section{Experimental protocol}

A total of ten groups with eight rats per group was employed in the present study.

Group I. Normal control: In the normal control group, the animals were kept in a cage for $12 \mathrm{~h}$ and were undisturbed except necessary procedure i.e., cleaning of the cage. Thereafter, the blood sample and myocardial tissue were collected as described earlier on the same day. The levels of CKMB, cTnI, IL-6 and IL10 were measured in the serum. The expression levels of HMGB1, GSK-3 $\beta$ and p-GSK-3 $\beta$ were assessed in the myocardial tissue homogenate.

Group II. Sham control group: In the sham-operated group, rats underwent laparotomy procedures only, without cecum ligation and puncture. After $12 \mathrm{~h}$ of laparotomy procedure, the blood sample and myocardial tissue were collected as described earlier. The different tests were performed as described in group I.

Group III. Sepsis group: Rats underwent a CLP procedure. After $12 \mathrm{~h}$ of laparotomy procedure, the blood sample and myocardial tissue were collected and the different tests were performed as described in group I.

Group IV. Telmisartan ( $2 \mathrm{mg} / \mathrm{kg}$ i.p.) in sepsis: Telmisartan (2 $\mathrm{mg} / \mathrm{kg}$ i.p.) was administered one hour after the CLP procedure in rats. After $12 \mathrm{~h}$ of laparotomy procedure, the blood sample and myocardial tissue were collected and different tests were performed as described in group I.

Group V. Telmisartan (4 mg/kg i.p.) in sepsis: Telmisartan (4 $\mathrm{mg} / \mathrm{kg}$ i.p.) was administered one hour after the CLP procedure in rats. After $12 \mathrm{~h}$ of laparotomy procedure, the blood sample and myocardial tissue were collected and different tests were performed as described in group I.

Group VI. AR-A014418 (1 mg/kg i.p.) in sepsis: AR-A014418 (1 $\mathrm{mg} / \mathrm{kg}$ i.p.) was administered one hour after the CLP procedure in rats. After $12 \mathrm{~h}$ of laparotomy procedure, the blood sample and myocardial tissue were collected and different tests were performed as described in group I.

Group VII. AR-A014418 (2 mg/kg i.p.) in sepsis-induced rats: AR-A014418 (3 mg/kg i.p.) was administered one hour after the CLP procedure in rats. After $12 \mathrm{~h}$ of laparotomy procedure, the blood sample and myocardial tissue were collected and the different tests were performed as described in group I.

Group VIII. Telmisartan per se group: Telmisartan $(4 \mathrm{mg} / \mathrm{kg}$ i.p.) was administered to normal rats. After $12 \mathrm{~h}$, the different tests were performed as described in group I.

Group IX. AR-A014418 per se group: AR-A014418 (2 mg/kg i.p.) was administered to normal rats. After $12 \mathrm{~h}$, the different tests were performed as described in group I.

Group X. DMSO in the sepsis group: 10\% DMSO dissolved in normal saline (vehicle for AR-A014418 and telmisartan) was administered $(0.2 \mathrm{ml} / 100 \mathrm{~g})$ one hour after the CLP procedure in rats. After $12 \mathrm{~h}$ of laparotomy procedure, the blood sample and myocardial tissue were collected and different tests were per- formed as described in group I.

\section{Statistical analysis}

In the present study, statistical analysis was performed using Graph Pad Prism (GraphPad Software Inc., La Jolla, CA, USA). One-way analysis of variance (ANOVA) followed by post-hoc Tukey's test was used for comparing the statistical differences among different groups. The results were expressed in the form of mean \pm standard deviation and the value $\mathrm{p}<0.05$ was considered to statistically significant.

\section{RESULTS}

Vehicle and per se administration of pharmacological agents did not affect sepsis-induced alterations in CK-MB, cTnI, HMGB1, IL-6, IL-10, and p-GSK-3 $\beta /$ GSK-3 $\beta$ ratio in a significant manner. Further, no significant effects on different parameters were observed in the sham control group in comparison to normal.

\section{Effect of cecal ligation and puncture-induced sepsis on biochemical parameters}

In the present study, cecal ligation and puncture significantly increased the myocardial injury assessed in terms of excess release of CK-MB and CTnI in the blood following $12 \mathrm{~h}$ of laparotomy procedure as compared to normal control (Figs. 1-3). Also, a significant increase in protein expression levels of HMGB1 and decreased phosphorylation of GSK-3 $\beta$ was observed in the myocardial tissue as compared to normal control (Figs. 4 and 5).

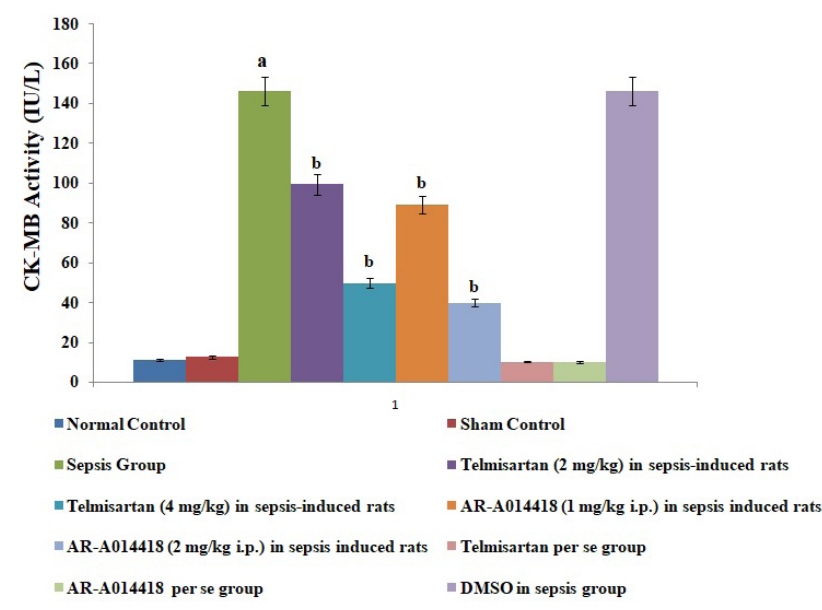

Fig. 1. Effects of cecal ligation and puncture-induced sepsis and pharmacological agents on creative kinase-MB (CK-MB) levels in the blood. Values are expressed as mean \pm standard deviation with $n$ $=8 .{ }^{a} p<0.05$ as compared to normal control; ${ }^{b} p<0.05$ as compared to sepsis control. 


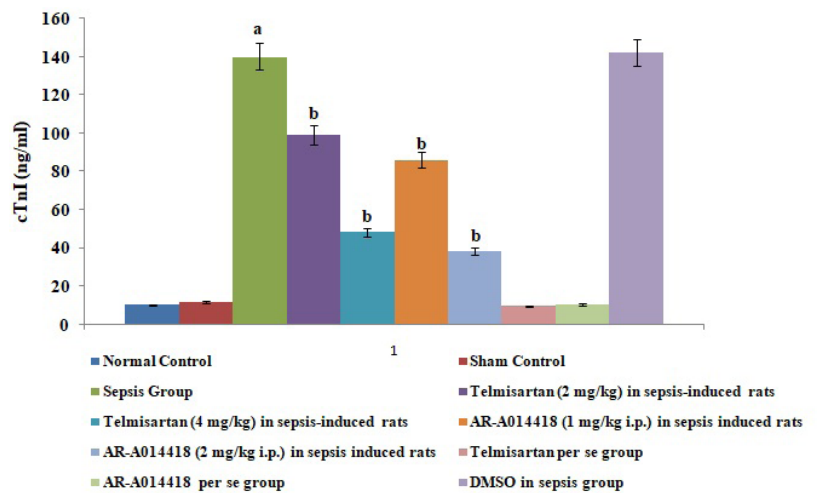

Fig. 2. Effects of cecal ligation and puncture-induced sepsis and pharmacological agents on cardiac troponin I (cTnl) levels in the blood. Values are expressed as mean \pm standard deviation with $n=8$. ${ }^{a} p<0.05$ as compared to normal control; ${ }^{b} p<0.05$ as compared to sepsis control.

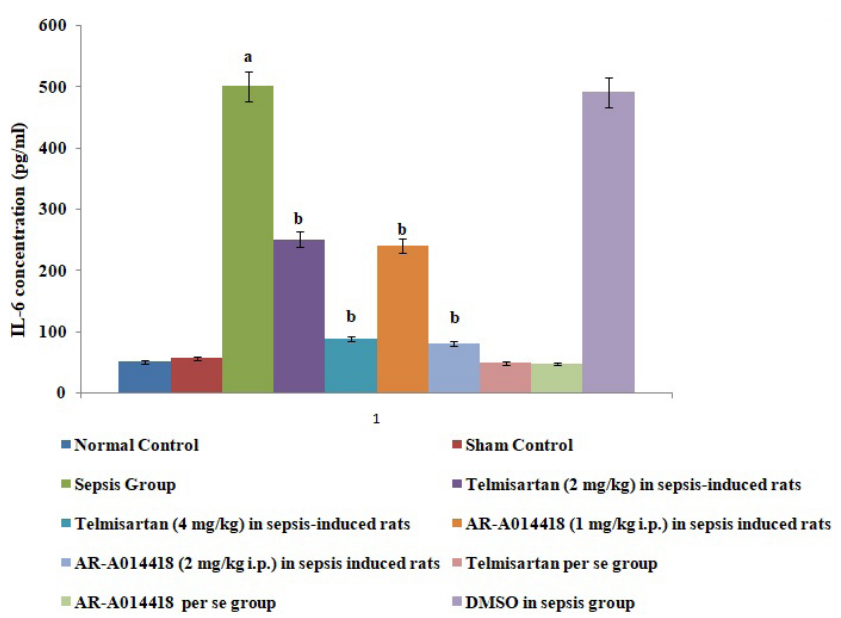

Fig. 3. Effects of cecal ligation and puncture-induced sepsis and pharmacological agents on interleukin (IL)- 6 levels in the blood. Values are expressed as mean \pm standard deviation with $n=8$. ${ }^{\mathrm{a}} \mathrm{p}<0.05$ as compared to normal control; ${ }^{b} \mathrm{p}<0.05$ as compared to sepsis control.

A significant increase in IL-6 and a decrease in IL-10 levels were noted in the blood at $12 \mathrm{~h}$ after cecal ligation and puncture as compared to normal control (Fig. 6).

\section{Effect of telmisartan and AR-A014418 on CLP-induced myocardial injury}

A single administration of telmisartan ( 2 and $4 \mathrm{mg} / \mathrm{kg}$ ) substantially reduced sepsis-induced myocardial injury in terms of decrease release of CK-MB and CTnI in the blood in sepsis- subjected rats, signifying the role of Ang II in mediating the sepsisinduced myocardial injury. Additionally, a single administration of AR-A014418 (1 and $2 \mathrm{mg} / \mathrm{kg}$ ) also considerably improved the myocardial injury by attenuating sepsis-induced alterations in sepsis-subjected animals, suggesting the involvement of GSK-3 $\beta$ kinase in sepsis-induced myocardial dysfunction. The effects of

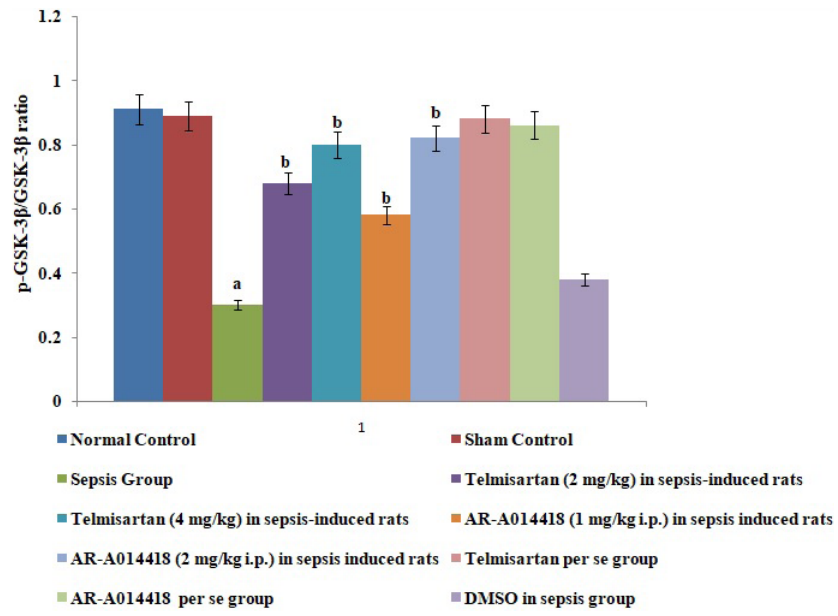

Fig. 4. Effects of cecal ligation and puncture-induced sepsis and pharmacological agents on $\mathrm{p}-\mathrm{GSK}-3 \beta / \mathrm{GSK}-3 \beta$ ratio in the myocardial tissue. Values are expressed as mean \pm standard deviation with $\mathrm{n}=8$. GSK-3 $\beta$, glycogen synthase kinase- $3 \beta .{ }^{\mathrm{a}} \mathrm{p}<0.05$ as compared to normal control; ${ }^{b} \mathrm{p}<0.05$ as compared to sepsis control.

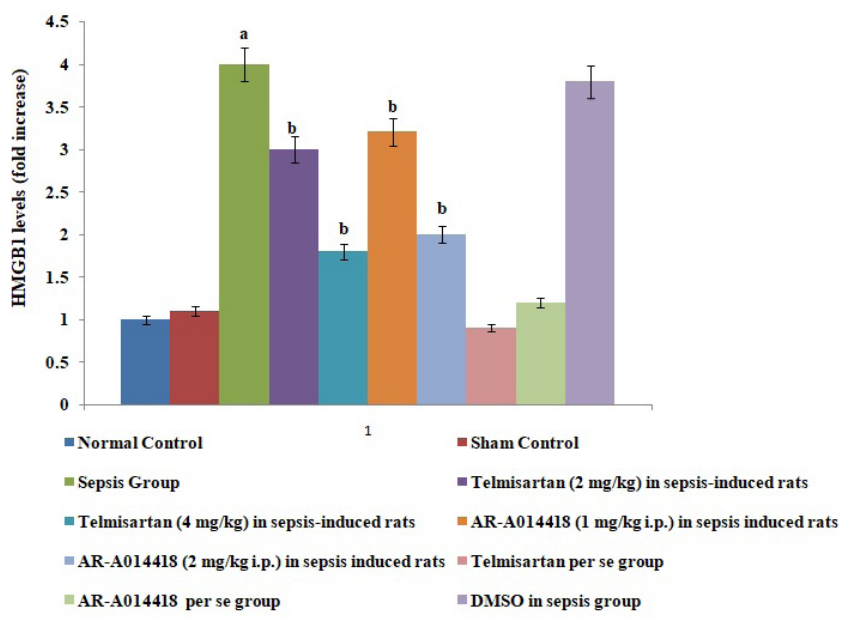

Fig. 5. Effects of cecal ligation and puncture-induced sepsis and pharmacological agents on high-mobility group box 1 (HMGB1) protein expression in the myocardial tissue. Values are expressed as mean \pm standard deviation with $n=8 .{ }^{a} \mathrm{p}<0.05$ as compared to normal control; ${ }^{\mathrm{b}} \mathrm{p}<0.05$ as compared to sepsis control.

telmisartan at dose $4 \mathrm{mg} / \mathrm{kg}$ and AR-A014418 at a dose of $2 \mathrm{mg} /$ $\mathrm{kg}$ were significantly higher than the telmisartan at a dose of 2 $\mathrm{mg} / \mathrm{kg}$ and AR-A014418 $1 \mathrm{mg} / \mathrm{kg}$ respectively (Figs. 1 and 2).

\section{Effect of telmisartan and AR-A014418 on CLP-induced inflammation parameters}

A single administration of telmisartan ( 2 and $4 \mathrm{mg} / \mathrm{kg}$ ) markedly decreased sepsis-induced inflammation in terms of decrease in IL-6 release in blood, decrease in protein HMGB1 expression in myocardial tissue and increase in IL-10 in the blood in sepsissubjected animals, signifying that Ang II mediates the inflam- 


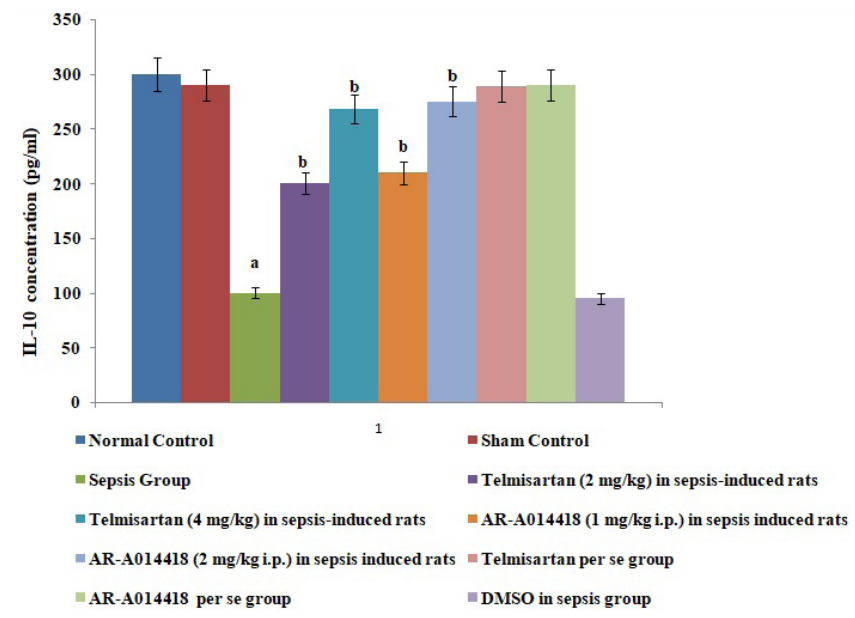

Fig. 6. Effects of cecal ligation and puncture-induced sepsis and pharmacological agents on interleukin (IL)-10 levels in the blood. Values are expressed as mean \pm standard deviation with $n=8$. ${ }^{\mathrm{a}} \mathrm{p}<0.05$ as compared to normal control; ${ }^{b} \mathrm{p}<0.05$ as compared to sepsis control.

mation in sepsis-induced injury via HMGB1, Il-6, and IL-10. Furthermore, a single administration of AR-A014418 (1 and 2 $\mathrm{mg} / \mathrm{kg}$ ) significantly improved the myocardial injury by attenuating sepsis-induced inflammation assessed in terms of decrease in IL-6, HMGB1 and increase in IL-10 (Figs. 3, 5, and 6). It suggests that CLP-induced sepsis may increase the GSK-3 $\beta$ activity, which further activates the HMGB1/RAGE axis to promote inflammation and myocardial injury in sepsis-subjected rats. The effects of telmisartan at dose $4 \mathrm{mg} / \mathrm{kg}$ and AR-A014418 at a dose of $2 \mathrm{mg} /$ $\mathrm{kg}$ were significantly higher than the telmisartan at a dose of 2 $\mathrm{mg} / \mathrm{kg}$ and AR-A014418 $1 \mathrm{mg} / \mathrm{kg}$, respectively.

\section{Effect of telmisartan and AR-A014418 on p-GSK-3 $\beta$ and GSK-3 $\beta$ levels}

A single administration of telmisartan (2 and $4 \mathrm{mg} / \mathrm{kg})$ significantly restored sepsis-induced decrease in p-GSK- $3 \beta /$ GSK- $3 \beta$ ratio in sepsis-subjected rats, suggesting that Ang II activates GSK$3 \beta$ to promote inflammation and myocardial injury in sepsisinduced rats. Similarly, a single administration of AR-A014418 (1 and $2 \mathrm{mg} / \mathrm{kg}$ ) significantly improved sepsis-induced decrease in p-GSK- $3 \beta /$ GSK- $3 \beta$ ratio signifying that increase in GSK-3 $\beta$ activity is potentially involved in the development of myocardial sepsis. The effects of telmisartan and AR-A014418 at dose 4 and 2 $\mathrm{mg} / \mathrm{kg}$ were significantly higher than at a dose of 2 and $1 \mathrm{mg} / \mathrm{kg}$, respectively (Fig. 4).

\section{DISCUSSION}

In the present study, CLP led to significant development of myocardial injury in terms of an increase in biochemical parameters viz. CK-MB and cTnI in the blood following $12 \mathrm{~h}$ of ligation puncture. CK-MB and cTnI are cardiac injury parameters and are often employed to assess myocardial injury in several studies $[18,19]$. CLP is one of the most common methods to induce myocardial sepsis [12] and the result of the present study showing myocardial injury in response to CLP is consistent with previous studies [20-22].

In the present study, CLP also led to increase in the inflammatory markers in the rats as assessed by an increase in levels of IL- 6 and a decrease in levels of IL-10 in the serum in CLPsubjected rats. IL-6 is a well documented pro-inflammatory cytokine $[19,21,22]$ and IL-10 is an anti-inflammatory cytokine [13]. Therefore, an increase in IL-6 and a decrease in IL-10 signifies the onset of inflammation in response to CLP. These results are inconsistent with previous studies $[23,24]$. Furthermore, there was an increase in the protein expression of HMGB1 in the hearts of CLP-subjected rats. HMGB1 is an intracellular protein, which acts as an inflammatory cytokine when released into the extracellular milieu. This extracellular HMGB1 causes multiple organ failure and is considered as a lethal mediator of sepsis [25]. Further, it has been reported that HMGB1 acts as an early mediator of inflammation and organ damage in ischemia-reperfusion injury [9]. Moreover, studies have demonstrated HMGB1 as an advanced marker of sepsis [26] and treatment with an antiHMGB1 antibody is more effective in sepsis rather than the treatment with anti-TNF- $\alpha$ and anti-IL- 6 antibodies [10]. HMGB1 exerts proinflammatory actions by mainly binding to RAGE. The involvement of the HMGB1-RAGE axis is implicated in many inflammatory heart disorders [16,17]. However, the mechanism of inflammatory sepsis-induced cardiac injury is not fully understood.

In the present study, the administration of telmisartan led to a significant alteration of CLP-induced myocardial injury assessed in terms of a decrease in CK-MB and CTnI in blood. Telmisartan is an $\mathrm{AT}_{1}$ antagonist which significantly inhibits the actions of Ang II. In the present study, telmisartan treatment also decreased the levels of IL-6, HMGB1 protein expression and increases the levels of IL-10 in sepsis-induced rats, suggesting the anti-inflammatory actions of telmisartan in sepsis-subjected rats. Clinical studies have also shown that telmisartan significantly inhibits the HMGB1/RAGE axis in stroke and hypertensive patients [15,16]. The inhibition of the HMGB1/RAGE axis has been proven as an effective and novel therapeutic strategy for treating several inflammatory disorders. However, it is the first report suggesting that telmisartan significantly attenuates sepsis-induced myocardial injury via inhibition of the HMGB1/RAGE axis. It is possible to suggest that CLP-induced sepsis may increase the levels of Ang II, which further activates the HMGB1/RAGE axis to promote the myocardial injury in sepsis-subjected rats.

In the present study, a significant decrease in the p-GSK-3 $\beta$ levels was observed in sepsis-induced rats, suggesting the increase in GSK-3 $\beta$ activity. Thus, it is possible to suggest that an increase in GSK-3 $\beta$ may also be involved in myocardial sepsis in rats. To fur- 
ther support this contention, GSK-3 $\beta$ inhibitor, AR-A014418 was employed in the present study. AR-A014418 is a selective GSK$3 \beta$ inhibitor and it has been shown that AR-A014418 treatment significantly protects the murine heart against the ischemiareperfusion injury by inhibiting the activity of GSK-3 $\beta$ [16]. In the present study, it was observed that AR-A014418 inhibits CLPinduced myocardial sepsis, suggesting that an increase in GSK-3 $\beta$ activity may be involved in the development of myocardial sepsis. AR-A014418 treatment also significantly decreased the levels of IL-6 and HMGB1, while it increased the levels of IL-10 in sepsisinduced rats. To best of our knowledge, it is the first report suggesting that AR-A014418 attenuates sepsis-induced myocardial injury via inhibition of the HMGB1/RAGE axis. It is possible to suggest that CLP-induced sepsis may increase the GSK-3 $\beta$ activity, which further activates the HMGB1/RAGE axis to promote inflammation and myocardial injury in sepsis-subjected rats.

In the present study, the administration of telmisartan significantly increased the p-GSK-3 $\beta$ levels suggesting the inhibition of GSK-3 $\beta$ activity in the presence of angiotensin receptor blockers. Other studies have also documented that Ang II increases GSK$3 \beta$ activity [27]. Accordingly, it is possible to suggest that GSK-3 $\beta$ is a downstream mediator of Ang II and an increase in the levels of Ang II may activate GSK- $3 \beta$ to promote inflammation and myocardial injury in sepsis-subjected rats.

Telmisartan and AR-A014418 have the potential to attenuate sepsis-induced myocardial injury. Telmisartan attenuates sepsisinduced myocardial injury via inhibition of HMGB1/RAGE axis and GSK-3 $\beta$ activity. Sepsis may increase the levels of Ang II to trigger GSK-3 $\beta$-dependent signaling to activate the HMGB1/ RAGE, which may promote inflammation and myocardial injury in sepsis-subjected rats.

\section{ACKNOWLEDGEMENTS}

The authors are grateful to the Emergency Department, Affiliated Hospital of Shandong University of Traditional Chinese Medicine, No. 16369, Jingshi Road, Lixia District, Jinan, Shandong Province, 2500014, China.

\section{CONFLICTS OF INTEREST}

The authors declare no conflicts of interest.

\section{REFERENCES}

1. Sagy M, Al-Qaqaa Y, Kim P. Definitions and pathophysiology of sepsis. Curr Probl Pediatr Adolesc Health Care. 2013;43:260-263.

2. Walley KR. Sepsis-induced myocardial dysfunction. Curr Opin Crit Care. 2018;24:292-299.
3. Wang Z, Bu L, Yang P, Feng S, Xu F. Alleviation of sepsis-induced cardiac dysfunction by overexpression of Sestrin 2 is associated with inhibition of p-S6K and activation of the p-AMPK pathway. Mol Med Rep. 2019;20:2511-2518.

4. Angus DC, Linde-Zwirble WT, Lidicker J, Clermont G, Carcillo J, Pinsky MR. Epidemiology of severe sepsis in the United States: analysis of incidence, outcome, and associated costs of care. Crit Care Med. 2001;29:1303-1310.

5. Sifi A, Adi-Bessalem S, Laraba-Djebari F. Role of angiotensin II and angiotensin type-1 receptor in scorpion venom-induced cardiac and aortic tissue inflammation. Exp Mol Pathol. 2017;102:32-40.

6. Wang H, Ward MF, Sama AE. Novel HMGB1-inhibiting therapeutic agents for experimental sepsis. Shock. 2009;32:348-357.

7. Tieu BC, Lee C, Sun H, Lejeune W, Recinos A 3rd, Ju X, Spratt H, Guo DC, Milewicz D, Tilton RG, Brasier AR. An adventitial IL-6/ MCP1 amplification loop accelerates macrophage-mediated vascular inflammation leading to aortic dissection in mice. J Clin Invest. 2009;119:3637-3651.

8. Xu J, Carretero OA, Liao TD, Peng H, Shesely EG, Xu J, Liu TS, Yang JJ, Reudelhuber TL, Yang XP. Local angiotensin II aggravates cardiac remodeling in hypertension. Am J Physiol Heart Circ Physiol. 2010;299:H1328-H1338.

9. Tsung A, Sahai R, Tanaka H, Nakao A, Fink MP, Lotze MT, Yang H, Li J, Tracey KJ, Geller DA, Billiar TR. The nuclear factor HMGB1 mediates hepatic injury after murine liver ischemia-reperfusion. $J$ Exp Med. 2005;201:1135-1143.

10. Bangert A, Andrassy M, Müller AM, Bockstahler M, Fischer A, Volz CH, Leib C, Göser S, Korkmaz-Icöz S, Zittrich S, Jungmann A, Lasitschka F, Pfitzer G, Müller OJ, Katus HA, Kaya Z. Critical role of RAGE and HMGB1 in inflammatory heart disease. Proc Natl Acad Sci U S A. 2016;113:E155-E164.

11. Volz HC, Kaya Z, Katus HA, Andrassy M. The role of HMGB1/ RAGE in inflammatory cardiomyopathy. Semin Thromb Hemost. 2010;36:185-194.

12. Wen H. Sepsis induced by cecal ligation and puncture. Methods Mol Biol. 2013;1031:117-124.

13. Faix JD. Biomarkers of sepsis. Crit Rev Clin Lab Sci. 2013;50:23-36.

14. Takahashi-Yanaga F. Roles of glycogen synthase kinase-3 (GSK-3) in cardiac development and heart disease. J UOEH. 2018;40:147-156.

15. Wei D, Xu H, Gai X, Jiang Y. Astragaloside IV alleviates myocardial ischemia-reperfusion injury in rats through regulating PI3K/AKT/ GSK-3 $\beta$ signaling pathways. Acta Cir Bras. 2019;34:e201900708.

16. Wong R, Aponte AM, Steenbergen C, Murphy E. Cardioprotection leads to novel changes in the mitochondrial proteome. Am J Physiol Heart Circ Physiol. 2010;298:H75-H91.

17. Wichterman KA, Baue AE, Chaudry IH. Sepsis and septic shock--a review of laboratory models and a proposal. J Surg Res. 1980;29:189201.

18. Kikuchi K, Tancharoen S, Ito T, Morimoto-Yamashita Y, Miura N, Kawahara K, Maruyama I, Murai Y, Tanaka E. Potential of the angiotensin receptor blockers (ARBs) telmisartan, irbesartan, and candesartan for inhibiting the HMGB1/RAGE axis in prevention and acute treatment of stroke. Int J Mol Sci. 2013;14:18899-18924.

19. Nakamura K, Yamagishi S, Nakamura Y, Takenaka K, Matsui T, Jinnouchi Y, Imaizumi T. Telmisartan inhibits expression of a receptor for advanced glycation end products (RAGE) in angiotensinII-exposed endothelial cells and decreases serum levels of soluble 
RAGE in patients with essential hypertension. Microvasc Res. 2005;70:137-141.

20. Zhang S, Qi W, Wang F, Xu Y, Wang X. [Effects of carbon monoxide release molecule-2 on sepsis-induced myocardial dysfunction in rats]. Zhonghua Wei Zhong Bing Ji Jiu Yi Xue. 2019;31:1097-1101. Chinese.

21. Lu ZQ, Lu JX, Deng YJ. Glucocorticoids offer protection against myocardial injury in a murine model of sepsis. Int J Clin Exp Med. 2015;8:12211-12218.

22. Sharma HS, Das DK. Role of cytokines in myocardial ischemia and reperfusion. Mediators Inflamm. 1997;6:175-183.

23. Li X, Cheng Q, Li J, He Y, Tian P, Xu C. Significance of hydrogen sulfide in sepsis-induced myocardial injury in rats. Exp Ther Med. 2017;14:2153-2161.

24. O'Riordan CE, Purvis GSD, Collotta D, Chiazza F, Wissuwa B, Al Zoubi S, Stiehler L, Martin L, Coldewey SM, Collino M, Thiemer- mann C. Bruton's tyrosine kinase inhibition attenuates the cardiac dysfunction caused by cecal ligation and puncture in mice. Front Immunol. 2019;10:2129.

25. Wang H, Bloom O, Zhang M, Vishnubhakat JM, Ombrellino M, Che J, Frazier A, Yang H, Ivanova S, Borovikova L, Manogue KR, Faist E, Abraham E, Andersson J, Andersson U, Molina PE, Abumrad NN, Sama A, Tracey KJ. HMG-1 as a late mediator of endotoxin lethality in mice. Science. 1999;285:248-251.

26. Zhen J, Li L, Yan J. [Advances in biomarkers of myocardial injury in sepsis]. Zhonghua Wei Zhong Bing Ji Jiu Yi Xue. 2018;30:699-702. Chinese.

27. Xin Y, Bai Y, Jiang X, Zhou S, Wang Y, Wintergerst KA, Cui T, Ji H, Tan Y, Cai L. Sulforaphane prevents angiotensin II-induced cardiomyopathy by activation of Nrf2 via stimulating the Akt/GSK-3 $\beta$ / Fyn pathway. Redox Biol. 2018;15:405-417. 